\title{
Finding the Hidden Pattern of Credit Card Holder's Churn: A Case of China
}

\author{
Guangli Nie ${ }^{1}$, Guoxun Wang ${ }^{1,3}$, Peng Zhang ${ }^{1}$, Yingjie Tian ${ }^{1}$, and Yong Shi ${ }^{1,2, *}$ \\ ${ }^{1}$ Research Center on Fictitious Economy and Data Science, \\ CAS, Beijing 100190, China \\ ${ }^{2}$ College of Information Science and Technology, University of Nebraska at Omaha, \\ Omaha, NE 68182, USA \\ ${ }^{3}$ School of Computer science and information engineering, Henan University, \\ Kaifeng 475001, China \\ sdungl@163.com, wangguoxun06@mails.gucas.ac.cn, \\ zhangpeng04@gmail.com, tianyingjie1213@163.com, yshi@gucas.ac.cn
}

\begin{abstract}
In this paper, we propose a framework of the whole process of churn prediction of credit card holder. In order to make the knowledge extracted from data mining more executable, we take the execution of the model into account during the whole process from variable designing to model understanding. Using the Logistic regression, we build a model based on the data of more than 5000 credit card holders. The tests of model perform very well.
\end{abstract}

Keywords: credit card churn, data mining, business intelligence.

\section{Introduction}

With cost-cutting and intensive competitive pressure, nowadays more and more companies start to focus on Customer Relationship Management (CRM). The unknown future behaviors of the customers are quite important to CRM. It is of crucial importance to detect the customers' future decision then the company can take corresponding actions early [6]. The customers who stop using the products or services of a company are usually called churners. Finding the churners can help the companies to retain the customers. Gustafsson, Johnson, and Roos (2005) studied telecommunication services to examine the effects of customer satisfaction and behavior on customer retention [7]. Results indicated a need for CRM managers to determine customer satisfaction more accurately in order to reduce customer churn.

One of the major reasons for this is that it cost less to retain existing customers than to acquire new customers [15]. It costs up to five times as much to make a sale to a new customer as it does to make an additional sale to an existing customer[4][17]. It is thought that $20 \%$ of a company's customers can account for $80 \%$ of its business [11]. And, it is becoming more evident that the only way to retain customers who bring profit in this industry is not only to be customer-driven but also to focus on building long-term relationships.

\footnotetext{
* Corresponding author.
} 
Due to the development of information technology, many companies have accumulated a large amount of data. Analyzing the data stored in the database can help the managers make the right marketing decision and pinpoint the right customers to market.

It is a very good field to predict the churn of credit card holders as the data of credit cards is more accessible and the churn of credit card holders is serious. Several studies have proved the effectiveness of the power of customer retention in credit cards. Van den Poel and Lariviere (2004) calculated the financial impacts of one percent increase in customer retention rate [18]. A bank is able to increase its profits by $85 \%$ due to a $5 \%$ improvement in the retention rate [14].

The main purpose of this paper is to provide a framework of understanding the knowledge of the card holders' hidden pattern instead of providing a new data mining algorithm, focusing on the application of the churn prediction. From data preparation to useful knowledge, the application goal of churn prediction covers the whole process of this paper. In this paper, we introduce a way to execute churn prediction considering execution and effectiveness of the model.

The rest of the paper is organized as follows. The definition of churn is introduced in Section 2. Section 3 summarizes the algorithms building model and criteria evaluating model. The data used in the research is described in Section 4, and the modeling process is presented in Section 5. The conclusions are introduced in the last section.

\section{Definition of Churn}

Churn is defined differently in different fields. Churn refers to the customer shift from one service provider to another [10]. Many of the existing researches use the behaviors related to product and a threshold fixed by a business rule to define churn. Once the transactions of the customer is low than the threshold, the customer would be regarded as a churner [6]. Van den Poel and Larivi`ere (2004) defined a churner as someone who closed his accounts [18]. Buckinx and Van den Poel (2005) defined a partial defector as someone with the frequency of purchases below the average and the ratio of the standard deviation of the interpurchase time to the mean interpurchase time above the average [1]. Glady, N.(2008) defined a churner as a customer with less than 2500 Euros of assets (savings, securities or other kinds of products) at the bank[6].Scott A. Neslin regarded customer as a churn according to the propensity of customers to cease doing business with a company in a given time period [13].

Churn in the specific telecommunications industry is also a hot topic. The broad definition of churn is the action that a customer's telecommunications service is canceled which includes both service-provider initiated churn such as customer's account being closed because of payment default and customer initiated churn. In the telecommunication study, only customer initiated churn is considered and it is defined by a series of cancel reason codes. Examples of reason codes are: unacceptable call quality, more favorable competitor's pricing plan, misinformation given by sales, customer expectation not met, billing problem, moving, change in business, and so on [10].

The relationship between churn rate and average lifetime is also studied. If no new customers are acquired then the average lifetime of an existing customer is equal to 1 / c, where $\mathrm{c}$ is the annual churn rate [13]. The study of Gustafsson, Johnson, and Roos (2005) includes customer satisfaction (CSt), affective commitment (ACt), calculative 
commitment $(\mathrm{CCt})$, a situational trigger condition (STt), and a reactional trigger condition (RTt), all in time $t$, to predict churn in time $t+1\left(\mathrm{Churn}_{\mathrm{t}+1}\right)[7]$.

In our application, we define that the customer who do not do any transaction with the bank on his own initiative during the observation period (explained later) is a churner.

\section{Algorithms and Evaluation Criteria}

In order to predict the churn of the customer effectively, it is of crucial importance to build an effective model which fulfills evaluation criteria. To accomplish this, there exist a lot of predictive modeling techniques available. Researchers use a variety of "approaches" to develop churn models, described by a combination of estimation technique, variable selection procedure, time allocations to various steps in the modelbuilding process, and a number of variables included in the model [13]. These data mining algorithms can help to select variables and build model [9]. The techniques include GA, Regression, Neural Networks, Decision Tree, Markov Model, Cluster Analysis [8].

According to the research of John Hadden [8], regression and decision tree are the two most popular algorithms used in the research and perform well. Neslin categorized the approaches as "Logit," "Trees," "Novice," "Discriminant," and "Explain." After comparison they found that the Logit and Tree approaches perform the best and result in firm predictive ability, the Novice approach is associated with middle-of-the road predictive performance, while the Discriminant and Explain approaches are associated with lower predictive performance [13].

In this paper, we also use these two algorithms to predict the churn of credit card holder and give the threshold of the early-warning alert system.

After building a predictive model, marketers want to use these classification models to predict future behavior of the customers. It is essential to evaluate the performance of the classifier. PCC, ROC are usually used as criteria. PCC, also known as accuracy, is undoubtedly the most commonly used evaluation metric of a classifier. PCC computes the ratio of correctly classified cases to the total number of cases to be classified. The receiver operating curve (ROC) is a graphical plot of the sensitivity which is the number of true positives versus the total number of events and 1-specificity which is the number of true negatives versus the total number of non-events. The ROC can also be represented by plotting the fraction of true positives versus the fraction of false positives [3]. We will evaluate the model by these criterias.

\section{Research Data}

\subsection{Data Source}

A major China commercial bank provided the data for this study. The data is extracted from a data warehouse of that bank. All of the data is organized at the level of customer. No matter in which branch the customer open the account, the data warehouse can identify the customer by name and identification number of the customer. All of the customers in the warehouse are indexed by a unique customer number. The data 
warehouse records all the past change of the card. Take the balance of the card for example, once the balance of the card changed, there will be one more row to keep the new balance and the last balance is still kept.

The data warehouse is built in 2005, thus the data can track back to Jan 2005 . There are 60 million customers in the data warehouse. We sample randomly from the system and the time interval of data is from Jan 2005 through the end of performance period (i.e. censoring on April 30, 2008) or the end of the customer relationship (i.e. churn). This is only raw data and we will refine the data when we calculate the variables.

The data relates to all the aspects of the credit card holder including the personal information of the card holder, the basic information of the card, the detailed transaction information, the abnormal usage information of the card and so on. There are 9 tables related to credit card and the detailed tables are list in following table.

Table 1. The tables related to the credit card

\begin{tabular}{|c|c|c|}
\hline No. & Name & Description \\
\hline 1 & Daily balance of the card & The daily balance record of the credit card \\
\hline 2 & Information of the card & The basic information of the credit card \\
\hline 3 & $\begin{array}{l}\text { Table of the abnormal } \\
\text { usage }\end{array}$ & The record about abnormal usage of the card \\
\hline 4 & Table of limit usage rate & $\begin{array}{l}\text { The rate of credit limit utilization when first } \\
\text { time used }\end{array}$ \\
\hline 5 & Table of first time used & $\begin{array}{l}\text { The information about the first time use of the } \\
\text { card }\end{array}$ \\
\hline 6 & Table of revoking pay & $\begin{array}{l}\text { The information about the bank revokes the } \\
\text { pay of card }\end{array}$ \\
\hline 7 & Table of suspending pay & $\begin{array}{l}\text { The information the holders do not pay off on } \\
\text { time }\end{array}$ \\
\hline 8 & $\begin{array}{l}\text { Table of the transactions of } \\
\text { the cards }\end{array}$ & $\begin{array}{l}\text { The information of the transactions of the } \\
\text { holder }\end{array}$ \\
\hline 9 & Personal information & The detailed information of the card holder \\
\hline
\end{tabular}

Dealing with the time in right way is quite important in this research. The time interval used in a telecommunication is 9-month. This period make sure that there is more variation using the 9-month cumulative churn measure [7]. Bart Larivi ere(2004) dealt with the time by customer's lifecycle. We can see that dealing with the time issue properly is quite important in this churn application [12].

The final aim of the churn study is to predict what will happen to the customer in the future, thus we must split the time window into two phases i.e. the observation period and the performance period. In the observation period, we design variables to observe the transaction behaviors of a customer, and then we check whether the customer becomes a churner or not in the performance period. When we build model the independent variables $\left(\mathrm{X}_{1} \mathrm{X}_{2} \ldots \ldots\right)$ are calculated from the data happened during the observation period and the dependent variable(Y) are calculated from the data happened during the performance period. In order to avoid the affect of the festival or 
season, we set a whole year (12 months) as observation period (the interval between $\mathrm{t}_{1}$ and $t_{2}$ and a whole year (12 months) as performance period (the interval between $t_{2}$ and $t_{3}$ ). In our study the observation period is from Jan 1, 06 to Dec 1, 2006 and the performance period is from Jan 12007 to Dec 31, 2007. After the observation of a whole year, our model will predict whether the customer become a churner or not in the performance period. The observation period must be a whole one, so Customer $\mathrm{C}$ of Fig. 1 will not be used.

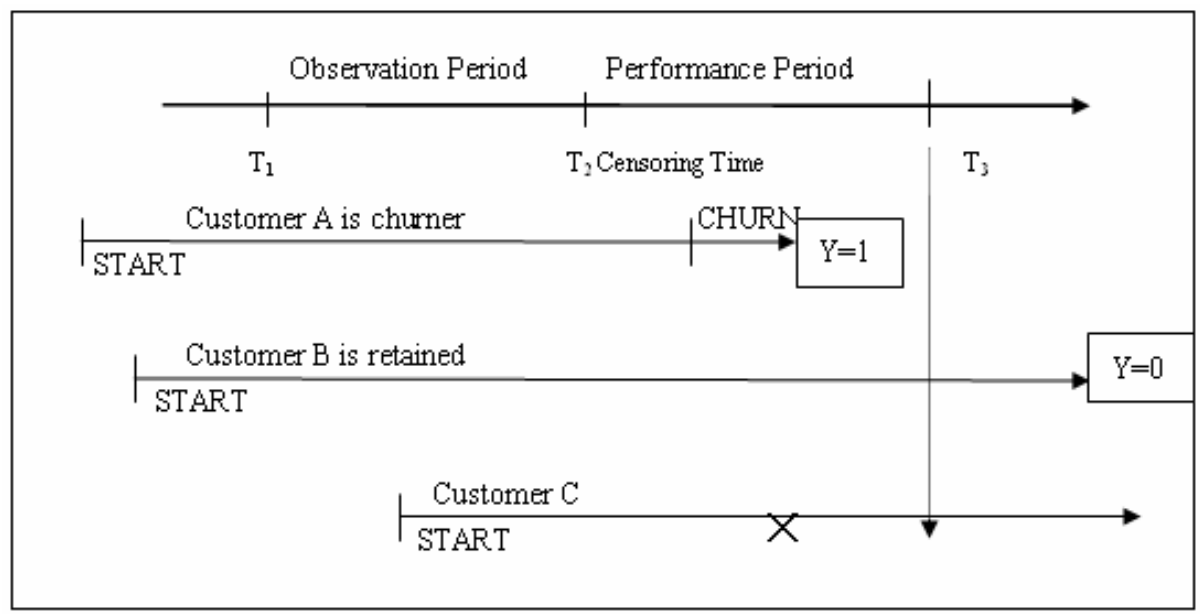

Fig. 1. The time window of analysis

\subsection{Variables Design}

Different types of the data may contribute differently to the model. The good derivable variables can help to build good model. Future researches could also study the types of data that are important for churn prediction models. For example, while the data includes behavioral, customer interaction, and demographic variables, it contains very little data which is directly useful for marketing. In different application, the input data varies. In a research of wireless industry, the input data are categorized in to four types: Demographics, Usage level, Quality of Service (QOS), Features/Marketing paging [17].

We do not give any assumption before data mining. We would not assume that some factors would affect the dependent variable while others do not affect in advance. The task of this phase is to design as many variables as possible. We designed the derivative variables from six perspectives: demographic variables, frequency variables, the total amount variables, time related variables, extreme variables and the status variables.

\subsection{Data Description}

The raw data we study consists of 9 tables and the largest table has 8 million records. The population consists of churner (without any activities during the performance 
period) and retained customers. All transactions are aggregated at the customer level. One customer may have several cards, but the object to predict is the customer, thus it is quite necessary to aggregate the data at the customer level. There are many methods to aggregate the data to customer level. One of the functions of the extreme variables is to aggregate the records to one as mentioned.

After the data preparation, we get a data mart consisting of 5456 samples, 440 are churners $(8.1 \%)$ and 5016 do not churn $(91.9 \%)$. Before building models, we separate the sample into training set to build the classifiers and a test set for the performance assessment.

According to the variable designed in Section 4.2, we calculate the derivative variables. There are 172 variables reflecting the complete information of the customer. The independent variables are calculated from the data during the observation period from January 2006 till December 2006 (12 months). The dependent variable (Y) is calculated from the data during the performance period from January 2007 till December 2007 (12 months). According the definition of Section 2, we define that the customer who did not do any transaction with the bank on his own initiative during the observation period is a churner.

$$
Y=\left\{\begin{array}{l}
1 \text { if customer has no transaction in performance period } \\
0 \text { if customer has at least one transaction in performance period }
\end{array}\right.
$$

\section{Modeling and Explanation}

The goal of this research is to show how to predict the churn of predict holder in an easy to execute and easy to understand way. Novel algorithm is not the aim of this research. The classifier applied in this research is well-known data mining algorithms: a logistic regression. This algorithm is quite mature and widely used. A lot of researches have proved the high accuracy and effectiveness of the algorithm [6] [8] [13].

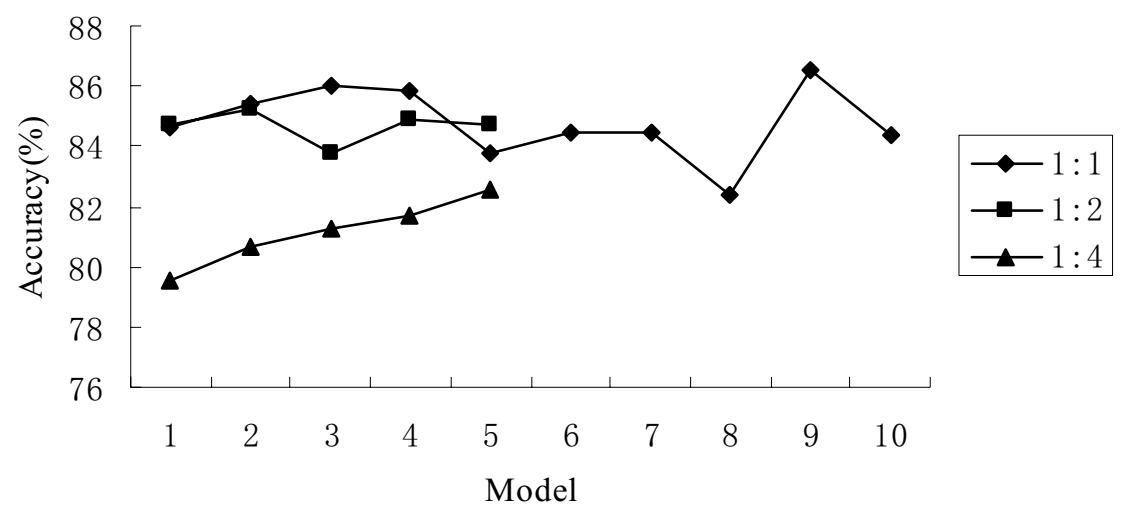

Fig. 2. The accuracy of the model with different proportion 
From last section, we can see that the proportion between current customers and churner of sample data is around 1: 9, which is unbalanced. In order to get the suitable proportion of the samples to build model, we try three proportions: 1:1, 1:2 and 1:4. The results of the proportions are list in the following Fig. 2. We can see that the proportion 1:1 performs the best and 1:4 perform worst. This means that the proportion $1: 1$ is the most suitable to existing data.

So we build ten models at the proportion 1:1 randomly sampling, which perform best. For the static churn model, a split sample design is used. Fifty percent of the churner (220 samples) and five percent of the non-churner (270 samples) is used to train the model; all of the rest (about 4960) is used to validate the model.

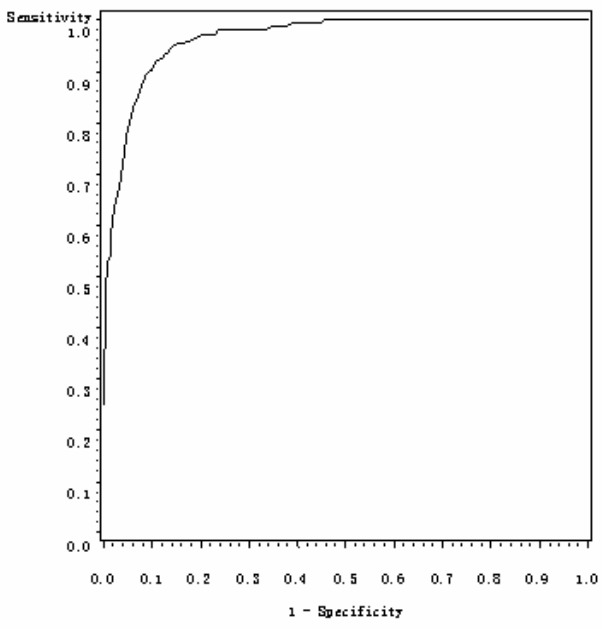

Fig. 3. The ROC curve of the classifier

The following rule reflects the hidden pattern of the credit card holder

$\mathrm{m}=-10.087+0.00705^{*}$ The proportion of overdraft time out of total time +1.6182 *The maximum length without action $+1.0594 *$ Whether the accountant being abnormal+3.6168*The times risk level higher than 3 recent 9 months+(-6.91E$07) *$ The number of cards callback+(-3.9418)*The length between issued and open of the cards $+(-0.00229) *$ The number of new cards in recent year+0.00573*The length between open and used for the first time of the cards $+0.035^{*}$ The length from last transaction of loan to now $+(-0.0814)^{*}$ The times expired of free interest $+3.117 *$ The loan times reason for deposit $+(-1.4932) *$ The proportion of the trade amount during National day out the whole month+(-10.087)*Whether VIP;

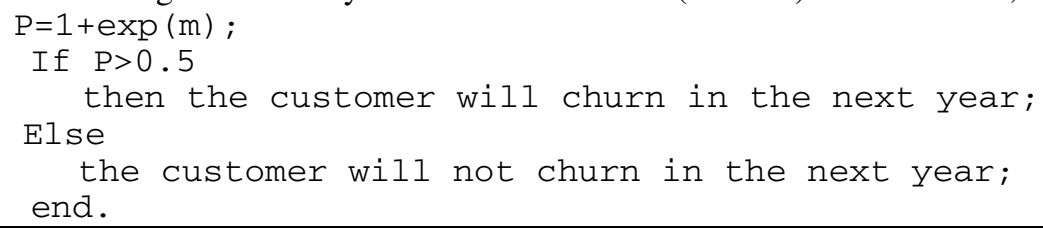


As introduced in the last section, the data mart consists of 172 variables. We used stepwise to reduce the variables. There are 13 variables chosen to build the model. The accuracy shown in Fig. 2 is the result of validation. We chose the best model as the final one. The ROC curve of the chosen model is shown in Fig. 3. The ROC curve shows that classifier performs very well.

The decision can be made based on above rule. Some of the variables are constant information such as the variable Whether VIP. This variable can only be used to predict the churn of the customer.

\section{Conclusions}

In this research, we propose the whole process of churn prediction of credit card and we demonstrate the whole process using a case of China. The purpose of this research is not to propose a new algorithm. The focus of this study is the execution and the understanding of the model. The suitable design of derivable variables and the right way to build model could be helpful to execute of the rule.

We split the time window into observation period and performance period. The independent variables are calculated from the data during the observation period, and the dependent variables are calculated from the data during the performance period.

After the data preparation, we get a data mart consisting of 5456 samples, 440 are churners $(8.1 \%)$ and 5016 do not churn (91.9\%). Based on the well-known data mining algorithms logistic regression, we build a very stable and accurate model. The variables are quite easy to conduct since we take the execution in account during the whole process.

\section{Acknowledgements}

The authors are very grateful to the anonymous bank that supplied the data to finish the analysis. This research has been partially supported by a grant from National Natural Science Foundation of China (\#70621001, \#70531040, \#70501030, \#70472074), Beijing Natural Science Foundation (\#9073020), 973 Project \#2004CB720103, Ministry of Science and Technology, China, and BHP Billiton Co., Australia.

\section{References}

1. Buckinx, W., Van den Poel, D.: Customer base analysis: Partial defection of behaviorallyloyal clients in a non-contractual fmcg retail setting. European Journal of Operational Research 164(1), 252-268 (2005)

2. Chiang, D.-A., Wang, Y.-F., Lee, S.-L., Lin, C.-J.: Goal-oriented sequential pattern for network banking churn analysis. Expert Systems with Applications 25(3), 293-302 (2003)

3. Coussement, K., van de Poel, D.V.: Churn prediction in subscription services: An application of support vector machines while comparing two parameter-selection techniques. Expert Systems with Applications 34, 313-327 (2008) 
4. Dixon, M.: 39 Experts Predict the Future. America's Community Banker 8(7), 20-31 (1999)

5. Floyd, T.: Creating a New Customer Experience. Bank Systems and Technology 37(1), R8-R13 (2000)

6. Glady, N., Baesens, B., Croux, C.: Modeling Churn Using Customer Lifetime Value. European Journal of Operational Research (2008), doi:10.1016/j.ejor.2008.06.027

7. Gustafsson, A., Johnson, M.D., Roos, I.: The effects of customer satisfaction, relationship commitment dimensions, and triggers on customer retention. Journal of Marketing 69(4), 210-218 (2005)

8. Hadden, J., Tiwari, A., et al.: Computer assisted customer churn management:State-of-theart and future trends. Computers \& Operations Research 34, 2902-2917 (2005)

9. Hung, S.-Y., Yen, D.C., Wang, H.-Y.: Applying data mining to telecom churn management. Expert Systems with Applications 31(3), 515-524 (2006)

10. Lu, J.: Predicting Customer Churn in the Telecommunications Industry - - An Application of Survival Analysis Modeling Using SAS. Sprint Communications Company

11. Kotler, P.: Marketing Management. Prentice-Hall, NJ (2000)

12. Lariviere, B., Van den Poel, D.: Investigating the role of product features in preventing customer churn, by using survival analysis and choice modeling: the case of financial services. Expert Systems with Applications 27, 277-285 (2004)

13. Neslin, S.A., Gupta, S., et al.: Defection Detection: Improving Predictive Accuracy of Customer Churn Models (2004)

14. Reichheld, F.F., Sasser Jr., W.E.: Zero defections: quality comes to service. Harvard Business Review 68(5), 105-111 (1990)

15. Roberts, J.H.: Developing New Rules for New Markets. Journal of the Academy of Marketing Science 28(1), 31-44 (2000)

16. Roos, I.: Switching Processes in Customer Relationships. Journal of Service Research 2, 76-93 (1999)

17. Slater, S.F., Narver, J.C.: Intelligence Generation and Superior Customer Value. Journal of the Academy of Marketing Science 28(1), 120-127 (2000)

18. Van den Poel, D., Larivi ere, B.: Customer attrition analysis for financial services using proportional hazard models. European Journal of Operational Research 157(1), 196-217 (2004)

19. Zhao, Y., Li, B., et al.: Customer Churn Prediction Using Improved One-Class Support Vector Machine ADMA (2005) 